\title{
Sequence-specific Detection of microRNAs by Signal-amplifying Ribozymes
}

Jörg S. Hartig, Imke Grüne, Saed H. Najafi-Shoushtari and Michael Famulok ${ }^{*}$

Kekulé Institut für Organische Chemie und Biochemie, Univ. Bonn, Gerhard-Domagk-Str.1, 53121 Bonn, Germany

\section{Supporting Information}

\section{Supplementary data 1: Multiple turnover}

Cleavage activity of HP-miR7 under multiple turnover conditions.

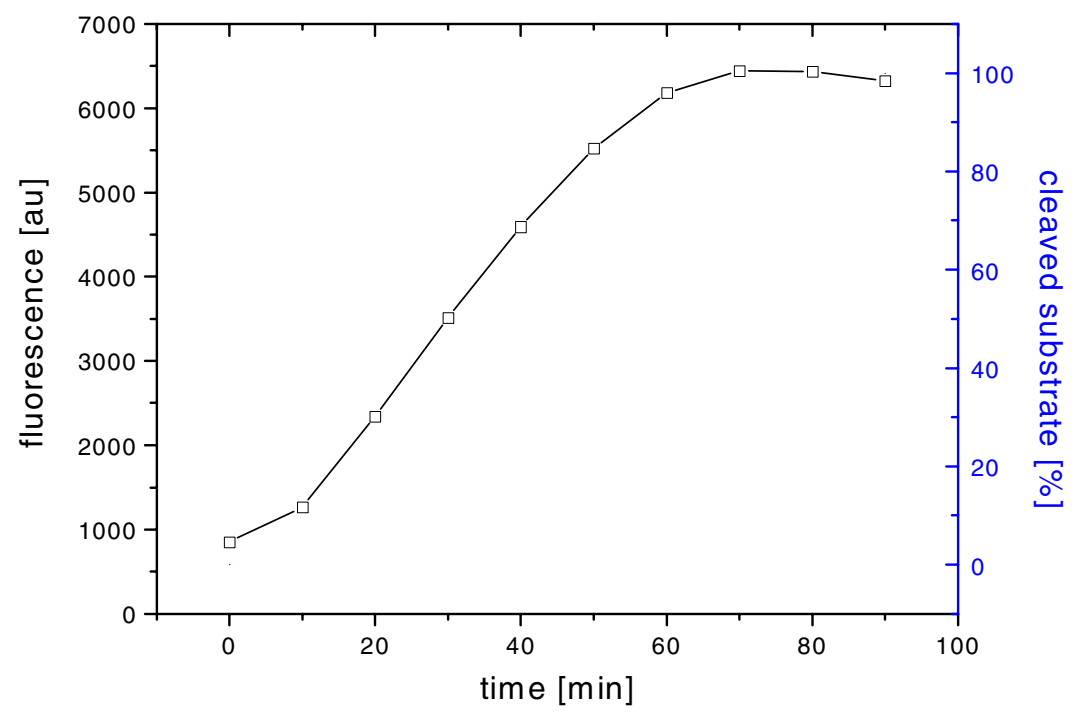

Reaction conditions: $10 \mathrm{nM}$ iHP-miR7, $10 \mathrm{nM}$ miR7, $200 \mathrm{nM}$ substrate, $50 \mathrm{mM}$ Tris $\mathrm{pH}$ 7.5, $30 \mathrm{mM} \mathrm{MgCl} 2$, volume $10 \mu \mathrm{L}$.

Conclusion: After 45 min: $~ 75 \%$ of the 2 pmoles of substrate is cleaved by 0.1 pmole ribozyme. Thus, the turnover number during the $45 \mathrm{~min}$ reaction is $15\left(0.33 \mathrm{~min}^{-1}\right)$. 


\section{Supplementary data 2: Oligodeoxyribonucleotides}

Primers and template used for PCR experiments and transcriptions:

HP-5'-P: 5'-TCT AAT ACG ACT CAC TAT AGG GAA ATA GAG AAG CGA-3' (italics: T7-promotor) HP-3'-P: 5'-TAC CAG GTA ATG TAC CAC GAC TTA CGT-3'

HP-let7: $\quad$ 5'-AAA TAG AGA AGC GAG CAC GAC TAT ACA ACC TAC TAC CTC TTC TCT ACG TGC ACC AGA GAA ACA CAC GAC GTA AGT CGT GGT ACA-3' (DNA-template)

5'-GGG AAA UAG AGA AGC GAG CAC GAC UAU ACA ACC UAC UAC CUC UUC UCU ACG UGC ACC AGA GAA ACA CAC GAC GUA AGU CGU GGU ACA UUA CCU GGU A-3' (RNA-sequence)

HP-miR1: 5'-AAA TAG AGA AGC GAG CAC GCA TAC TTC TTT ACA TTC CAT TCT CTA CGT GCA CCA GAG AAA CAC ACG ACG TAA GTC GTG GTA CA-3' (DNA-template)

5'-GGG AAA UAG AGA AGC GAG CAC GCA UAC UUC UUU ACA UUC CAU UCU CUA CGU GCA

CCA GAG AAA CAC ACG ACG UAA GUC GUG GUA CAU UAC CUG GUA -3' (RNA-sequence)

HP-miR2: 5'-AAA TAG AGA AGC GAG CAG CGC ACA TCA AAG CTG GCT TCT CTG CTG CAC CAG AGA AAC ACA CGA CGT AAG TCG TGG TAC A-3' (DNA-template)

5'-GGG AAA UAG AGA AGC GAG CAG CGC ACA UCA AAG CUG GCU UCU CUG CUG CAC CAG

AGA AAC ACA CGA CGU AAG UCG UGG UAC AUU ACC UGGU A -3' (RNA-sequence)

HP-miR4: 5'-GGG AAA TAG AGA AGC GAG CAC GCA ATG GTT GTC TAG CTT TAT TCT CTA CGT GCA CCA GAG AAA CAC ACG ACG TAA GTC GTG GTA CA-3' (DNA-template)

5'-GGG AAA UAG AGA AGC GAG CAC GCA AUG GUU GUC UAG CUU UAU UCU CUA CGU GCA

CCA GAG AAA CAC ACG ACG UAA GUC GUG GUA CAU UAC CUG GUA -3' (RNA-sequence)

HP-miR5: 5'-AAA TAG AGA AGC GAG CAC GCA TAT CAC AAC GAT CGT CCT TTT CTC TAC GTG CAC CAG AGA AAC ACA CGA CGT AAG TCG TGG TAC A-3' (DNA-template)

5'-GGG AAA UAG AGA AGC GAG CAC GCA UAU CAC AAC GAU CGU CCU UUU CUC UAC GUG

CAC CAG AGA AAC ACA CGA CGU AAG UCG UGG UAC AUU ACC UGG UA-3' (RNA-sequence)

HP-miR7: 5'-AAA TAG AGA AGC GAC GAG CAC AAC AAA ATC ACT AGT CTT CTC TAG CTC GAC CAG AGA AAC ACA CGA CGT AAG TCG TGG TAC A-3' (DNA-template)

5'-GGG AAA UAG AGA AGC GAC GAG CAC AAC AAA AUC ACU AGU CUU CUC UAG CUC GAC

CAG AGA AAC ACA CGA CGU AAG UCG UGG UAC AUU ACC UGG UA-3' (RNA-sequence)

HP-miR10: 5'-GGG AAA TAG AGA AGC GAG CTC GAC AAA TTC GGA TCT ACA CTT TTC TAC GAG CAC CAG AGA AAC ACA CGA CGT AAG TCG TGG TAC A-3' (DNA-template)

5'-GGG AAA UAG AGA AGC GAG CUC GAC AAA UUC GGA UCU ACA CUU UUC UAC GAG CAC

CAG AGA AAC ACA CGA CGU AAG UCG UGG UAC AUU ACC UGG UA-3' (RNA-sequence)

HP-miR34: 5'-AAA TAG AGA AGC GAC GTG CCA ACC AGC TAA CAC ACT GCC TTC TCT AGC ACG ACC AGA GAA ACA CAC GAC GTA AGT CGT GGT ACA-3' (DNA-template)

5'-GGG AAA UAG AGA AGC GAC GUG CCA ACC AGC UAA CAC ACU GCC UUC UCU AGC ACG

ACC AGA GAA ACA CAC GAC GUA AGU CGU GGU ACA UUA CCU GGU A-3' (RNA-sequence)

HP-miR79: 5'-GGG AAA TAG AGA AGC GAG CTG CGC TTT GGT AAT CTA GCT TCT CTA GCA GCA CCA GAG AAA CAC ACG ACG TAA GTC GTG GTA CA-3' (DNA-template)

5'-GGG AAA UAG AGA AGC GAG CUG CGC UUU GGU AAU CUA GCU UCU CUA GCA GCA CCA GAG AAA CAC ACG ACG UAA GUC GUG GUA CAU UAC CUG GUA -3' (RNA-sequence)

(bold: annealing region of the primer; blue/green: domain $\mathrm{C}$, blue: microRNA-annealing region, green: annealing region to domain $\mathrm{A}$ ). Underlined: the whole inserted domain $\mathrm{C}$ including the stem region (underlined black).

Molecular Beacon:

MB-miR7: 5'-FAM-GCG AGC AAC AAA ATC ACT AGT CTT CCC TCG C-Dabcyl-3' Oligoribonucleotides

Substrate for ribozyme reactions:

Substrate: 5'-Cy3-UCGCAGUCCUAUUUAUUG-FAM-3'

MicroRNA-sequences of drosophila melanogaster:

miR1: 5'-UGGAAUGUAAAGAAGUAUGGAG-3'

miR2a: 5'-UAUCACAGCCAGCUUUGAUGAGC-3' 
miR4: 5'-AUAAAGCUAGACAACCAUUGA-3'

miR5: 5'-AAAGGAACGAUCGUUGUGAUAUG-3'

miR7: 5'-UGGAAGACUAGUGAUUUUGUUGU-3'

miR10: 5'-ACCCUGUAGAUCCGAAUUUGU-3'

miR34: 5'-GGCAGUGUGGUUAGCUGGUUG-3'

miR79: 5'-AUAAAGCUAGAUUACCAAAGC-3'

let7: 5'-UGAGGUAGUAGGUUGUAUAGU-3'

Standard oligonucleotides and molecular beacon were purchased from MWG, Ebersberg, Germany. The labeled substrate and the microRNA-sequences were synthesized by Dharmacon, Lafayette, CO. The DNA template sequences for the ribozymes were PCR amplified with Taq DNA polymerase (Promega) and transcribed with T7 RNA polymerase (Stratagene). 


\section{Supplementary data 3: Molecular Beacon reaction}

Molecular beacon reactions were carried out in $50 \mathrm{mM}$ Tris/HCL pH 7.5 and $30 \mathrm{mM} \mathrm{MgCl}$.Molecular beacon and microRNA were incubated for $10 \mathrm{~min}$ at $37{ }^{\circ} \mathrm{C}$. The hybridization reaction was monitored by measurement of the fluorescence of the FAM-group with excitation at $485 \mathrm{~nm}$ and emission at $518 \mathrm{~nm}$ (Fluorscan Ascent FL). The test reaction was performed in a reaction volume of $50 \mu \mathrm{L}$ with $25 \mathrm{nM} \mathrm{MB}-\mathbf{m i R} 7$ and 25 to $200 \mathrm{nM}$ miR7. The reactions for the comparison of the beacon (figure4 shown in communication) with the ribozyme were performed in a reaction volume of $10 \mu \mathrm{L}$ with $10 \mathrm{nM}$ of MB-miR7 and of 1-20 $\mathrm{nM}$ of miR7.

\section{Molecular Beacon test reaction}

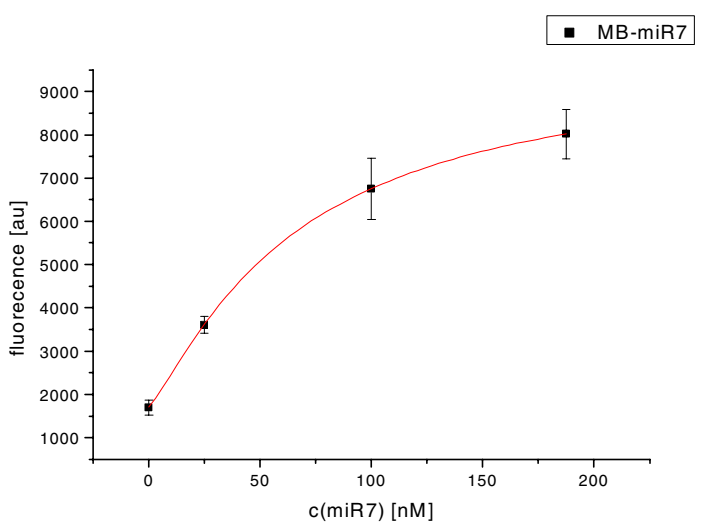

Fluorescence signal of the MB-miR7 in the presence of different miR7 concentrations. Reaction volume $50 \mu \mathrm{L}$, MBmiR7 $25 \mathrm{nM}$, miR7 25-200 nM.

Comparison of the sensitivity of ribozyme and beacon (at $200 \mathrm{nM}$ beacon, and $200 \mathrm{nM}$ ribozyme substrate, respectively, using 1-20 nM microRNA miR7 in $10 \mu \mathrm{L}$ reaction volume)

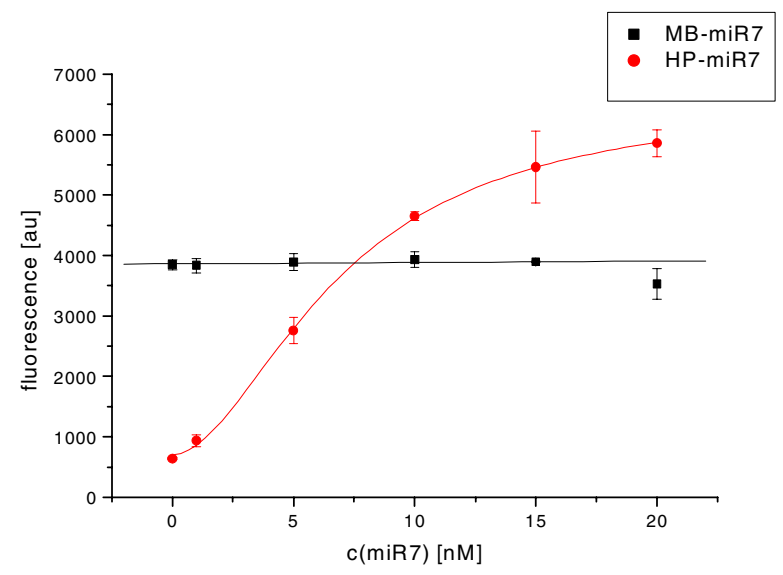

Comparison of MB-miR7 with HP-miR7. black: fluorescence of MB-miR7 (200 nM) and red: fluorescence of HP-miR7 (10 nM, $200 \mathrm{nM}$ substrate) at 1-20 nM miR7 (50 mM Tris pH 7.5, $30 \mathrm{mM} \mathrm{MgCl}_{2}$, volume: $\left.10 \mu \mathrm{L}\right)$. 


\section{Supplementary data 4: Ribozyme reactions}

Ribozyme reactions were carried out in $50 \mathrm{mM}$ Tris/HCL pH 7.5, $30 \mathrm{mM} \mathrm{MgCl} 2$ and $200 \mathrm{nM}$ (20-fold excess over ribozyme) of labeled substrate in 384-well-plates (Corning). Ribozyme, substrate and microRNA were incubated $10 \mathrm{~min}$ at $37{ }^{\circ} \mathrm{C}$. The reaction was started upon the addition of $\mathrm{MgCl}_{2}$. The cleavage reaction was monitored by measurement of the fluorescence of the FAM-group with excitation at $485 \mathrm{~nm}$ and emission at 518 nm (Fluorscan Ascent FL). The reaction conditions were the same as those in Figure 2.

\section{Supplementary data 5: Calibration curves}
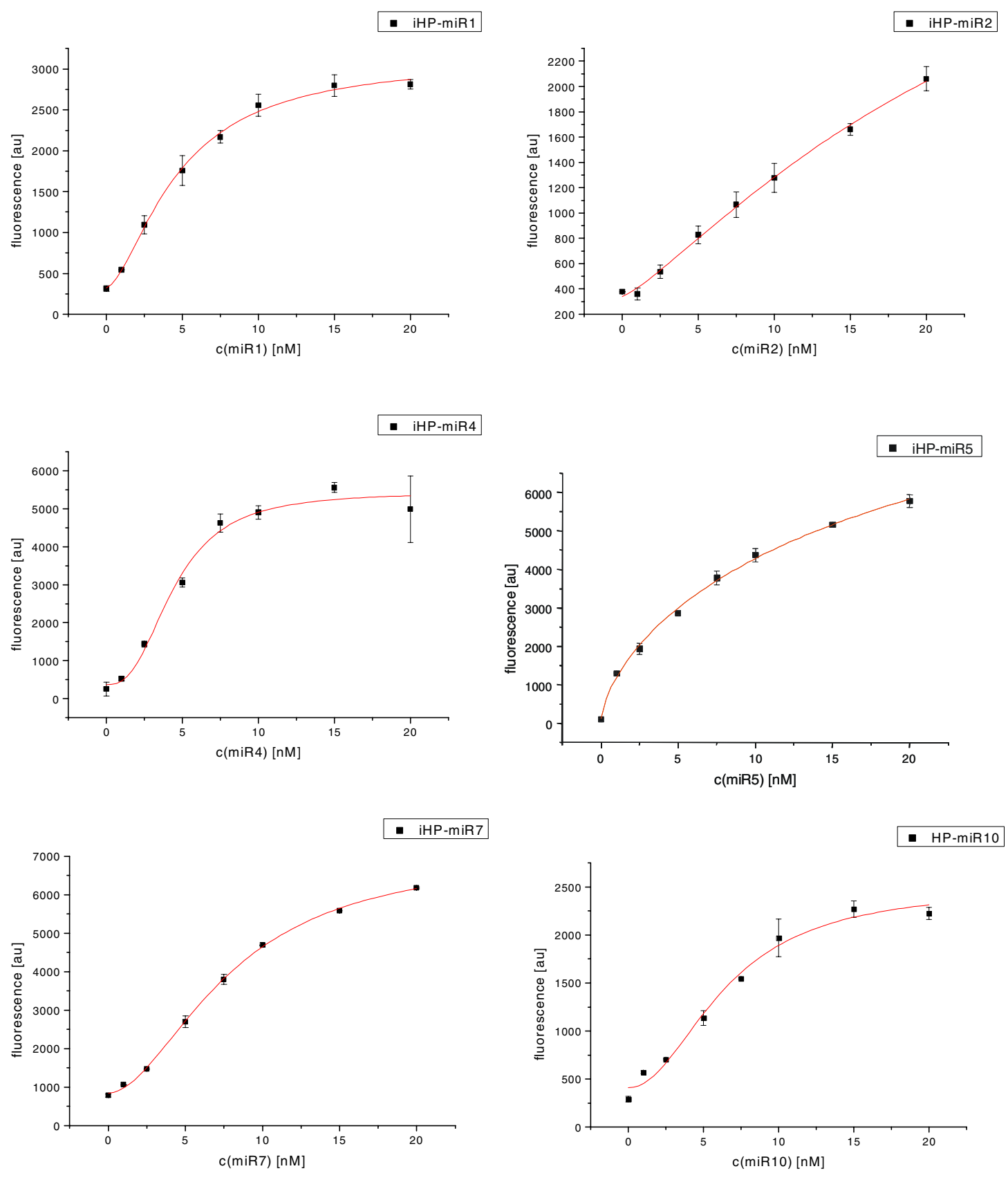

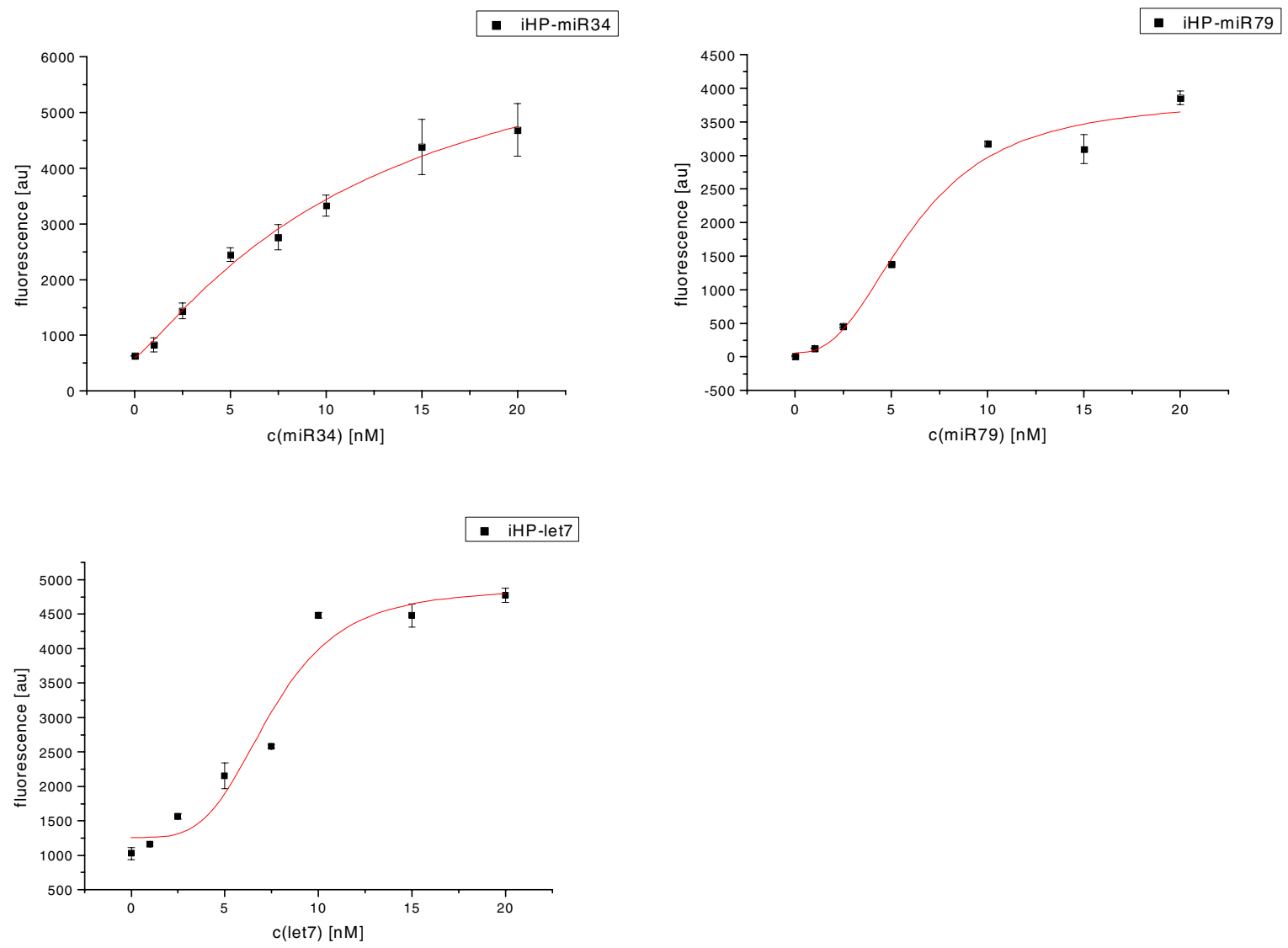

Cleavage activity of all 9 ribozymes in the presence of different microRNA concentrations. Reaction volume $10 \mu \mathrm{L}$, ribozyme $10 \mathrm{nM}$, microRNA 1-20 nM. 